\title{
Scolia
}

Revue de linguistique

\section{Le focus initial en français vernaculaire}

The Initial Focus in Vernacular French

\section{Pierre Larrivée}

\section{OpenEdition}

Journals

Édition électronique

URL : http://journals.openedition.org/scolia/1126

DOI : 10.4000/scolia. 1126

ISSN : 2677-4224

\section{Éditeur}

Presses universitaires de Strasbourg

\section{Édition imprimée}

Date de publication : 10 juillet 2020

Pagination : $33-50$

ISBN : 979-10-344-0067-6

ISSN : 1253-9708

\section{Référence électronique}

Pierre Larrivée, "Le focus initial en français vernaculaire », Scolia [En ligne], 34 | 2020, mis en ligne le 10 juillet 2020, consulté le 10 juillet 2020. URL : http://journals.openedition.org/scolia/1126 ; DOI : https://doi.org/10.4000/scolia.1126 
SCOLIA 34/2020, p. 33-50.

\title{
Le focus initial en français vernaculaire
}

\author{
Pierre LARRIVÉE \\ Université de Caen, CRISCO-EA 4255 \\ Pierre.Larrivee@unicaen.fr
}

\section{Introduction}

On suppose mutatis mutandis que les grandes catégories syntaxiques sont disponibles dans chaque langue. Pourtant, la façon dont ces catégories sont réalisées est susceptible de varier. Ainsi, la phrase assertive ordinaire du français vernaculaire actuel aurait la forme SVO, avec des sujets le plus souvent réalisés par des clitiques envisagés comme des marqueurs morphologiques (Legendre et Culbertson, 2008). Plus de $97 \%$ des propositions conjuguées en français vernaculaire correspondraient en effet au schéma Sujet clitique + Verbe (Meisner, 2010), validant l'idée de Lambrecht (1987) que Il parle serait syntaxiquement plus canonique que Paul parle. Les groupes à noyau nominal verraient leur réalisation déterminée par leur statut discursif. Les groupes nominaux représentant de l'information nouvelle semblent principalement introduits par la clivée présentative il y $a$, qui représente à peu près $5 \%$ des énoncés dans l'étude de corpus de Larrivée (2015). La même étude montre que les groupes nominaux exprimant de l'information ancienne sont représentés par des dislocations, présentes dans près de $10 \%$ des propositions; et enfin que plus de $3 \%$ des propositions sont introduites par la clivée en c'est... que, le groupe nominal qu'elle focalise oscillant entre valeur contrastive, ancienne et nouvelle. Ce profil explique sans doute l'évaluation proposée par certains chercheurs selon laquelle le français 
vernaculaire actuel est une langue discursive, dont la syntaxe marque la valeur d'information ancienne et nouvelle des constituants (De Cat, 2008; voir aussi Lambrecht, 1987).

Cette caractérisation du français comme langue discursive laisse attendre un ensemble de réalisations associées à des schémas récurrents. On suppose depuis l'hypothèse du CP éclaté de Rizzi (1997) que des projections associent des syntagmes à des valeurs discursives. Situées dans la périphérie gauche qui assure le lien avec le contexte antécédent, les projections de Topique et celle de Focus peuvent accueillir les différentes configurations à valeur d'information ancienne et nouvelle. Ceci étant, les nominativus pendens (1), les dislocations (2), les topicalisations (3) et le focus initial (4) ne sont pas tous réalisés avec la même fréquence dans toutes les langues.

1) Und Gesang - habt ihr denn hier irgendwie so einen Lehrer...?

(Nolda, 2013 : 429)

Et chanter - tu as un professeur?

2) Moi, les huîtres panées, ça m'écœure.

3) (She claims she speaks Basque,) and speak Basque she does. Elle disait qu'elle parlait basque, et effectivement, pour parler basque elle parle basque.

4) Cecilia ho invitato. (voir Bianchi, $2013: 193$ )

C'est Cécile que j'ai invitée.

La topicalisation du groupe verbal de l'anglais illustrée en (3), grossièrement traduite, n'a pas d'équivalent en français. De même, la focalisation initiale de l'italien, en (4), normalement traduite par la clivée en français, n'y en a pas moins un correspondant:

5) Cécile j’ai invité.

Dans ce cas, clairement, l'objet nominal qui devrait se trouver en position postverbale est déplacé en position initiale de Focus à cause de sa valeur informationnelle. Cependant, cette configuration semble limitée à la pratique vernaculaire du français de France et de Belgique, où elle semble rare.

Le propos de cet article est d'articuler la tension entre la disponibilité structurale d'une construction et des asymétries de fréquences. Nous examinons d'abord les propriétés structurales de la configuration à Focus initial. Les valeurs de la configuration sont ensuite étudiées à partir d'attestation et d'élicitations. L'explication fournie de la rareté 
relative de la configuration à Focus initial se fait en deux temps: premièrement, cette configuration est minorée par l'importance que prend la clivée en français; d'autre part, l'importance de la clivée et la rareté du Focus initial sont associées aux propriétés prosodiques de la phrase française, qui défavorise l'accentuation préverbale, suivant Lambrecht (1994).

\section{Focus initial}

Au-delà des relations intra-prédicatives, la recherche syntaxique a cherché à interroger l'organisation des syntagmes périphériques de la phrase. En effet, pose question tout un ensemble de phénomènes dont on commence à appréhender les contours. Se concentrant sur les groupes nominaux, on relève dans la littérature les termes d'adresse (Lagorgette, 2003):

6) - Ça va, Manu?

- Dégage, bolosse.

les nominativus pendens (ou Hanging Topics, Le Querler, 2003; Missire et Rouayrenc, 2014; Quignard et alii, 2016):

7) a. ces souliers j'écrase les pieds de tout le monde (Deulofeu, 1977)

b. - et sur la falaise quel est vraiment son travail

- alors son travail eh ben il pose des grillages (Blanche-

Benveniste, 1996 : 116)

les dislocations:

8) a. Mon frère sa moto le guidon il est cassé (Blasco, 1995)

b. Les enfants, leurs amis, les jouets, ils les prêtent pas facilement (Deulofeu, 1977)

les focus initiaux ${ }^{1}$ :

9) a. Une fortune ça m'a coûté! (Blanche-Benveniste, 2002: 111)

b. Rien que des pâtes ils mangent là-bas (Blanche-Benveniste, $1996: 114)^{2}$

1 Quoiqu'une occurrence comme la suivante ressemble plus à un Topique contrastif: Trois enfants j'ai eus, et tous les trois je les ai perdus (Blanche-Benveniste, 1996 : 112)

2 Un relecteur suggère une analyse alternative, que je rapporte ici pour mémoire; je ne l'adopte pas parce que le fait de traiter une proposition comme disloquée me semble singulier, et le syntagme initial n'a pas statut de dislocation, comme le montrent les études existantes résumées dans cette section. 
Un certain nombre de caractéristiques séparent ces différentes configurations. On peut se demander si le terme d'adresse (incluant les insultes), qui se retrouve en position gauche, médiane ou droite, fait partie de l'énoncé, où il ne peut jamais être repris par un pronom, ou constitue un énoncé à lui seul. On admet généralement que le nominativus pendens, à position initiale fixe, a un rapport avec la proposition $^{3}$, sans pourtant pouvoir être repris. Ce rapport se retrouve de même avec la dislocation et le focus initial, qui se distinguent sous les trois rapports de la liberté de position, de la reprise et de la valeur informationnelle.

\begin{tabular}{|l|c|c|c|c|}
\hline & $\begin{array}{c}\text { Interne à la } \\
\text { proposition }\end{array}$ & Position libre & Reprise & Topique \\
\hline Terme d'adresse & - & + & - & - \\
\hline Nominativus pendens & + & - & - & + \\
\hline Dislocation & + & + & + & + \\
\hline Focus initial & + & - & - & - \\
\hline
\end{tabular}

Tableau 1. Éléments périphériques de la proposition conjuguée

Considérons ces deux dernières catégories, illustrées respectivement par (10a) et (10b):

10) a. Cécile, je l'ai invitée.

b. Cécile j'ai invitée.

"Vous tenez pour acquis que dans [une fortune ça m'a coûté], le SN [une fortune] est un complément du verbe, placé en tête et ainsi focalisé. Mais une autre analyse est possible: que ce SN soit une P nominale, et que [ça m'a coûté] soit un ajout disloqué à droite de celle-ci. Cette analyse a l'avantage d'être compatible avec la structure prosodique: [une fortunel] porte un intonème conclusif, et [ça m'a coûté] porte un contour "bas plat" d'appendice, comme d'ordinaire les éléments disloqués à droite. Elle explique aussi naturellement pourquoi [une fortune] est focal, et pourquoi [ça m'a coûté] s'interprète comme substrat (au sens de Kronning), c'est-à-dire comme la confirmation d'une information implicite. Bref, tout cet article repose sur le postulat qu'il existe des "focus initiaux". Et s'il ne s'agissait en réalité que de "SV postfixés" ?»

3 Un relecteur demande opportunément quelle est la nature de ce rapport pour le hanging topic. Ce rapport n'étant pas le propos de ce travail, je renvoie, comme on me le suggère, au Groupe de Fribourg (2012: 173ss) pour une analyse énonciative; dans le cadre élaboré à partir de Rizzi (1997), le hanging topic se trouverait dans une position Topique haute. 
Les quelques études sur le Focus initial (Sabio, 1995; BlancheBenveniste, 1996; Rowlett, 1998: 130; Abeillé, Godard \& Sabio 2008a et $b$; Authier \& Haegeman, 2018) notent qu'il se distingue d'une construction intermédiaire représentée par (11).

11) a. Cécile, j’aime pas.

b. Cécile j'aime pas.

Dans ces deux configurations, le syntagme est initial. Il se distingue par l'intonation: une pause sépare le nom initial de la proposition en (11a), alors que l'intonation du Focus initial (11b) est continue avec un ton bas sur la proposition selon Blanche-Benveniste (voir Tanguy, 2010). L'élément initial peut être chevillé à la proposition par un que dans la construction à Focus (b), mais jamais en (a):

12) a. Cécile, $\left({ }^{*} q u e\right)$ j’aime pas.

b. Cécile que j'aime pas.

Elles se différencient par la reprise pronominale, qui est admise pour (11a) et exclue pour (11b):

13) a. Cécile, je l'aime pas.

b. Cécile (que) je (*l') aime pas.

C'est pourquoi on suppose que le cas en (a) est une dislocation sans pronom résomptif. Cette construction se retrouve généralement avec des noms à valeur générique, un sujet nominal ou pronominal et un ensemble limité de verbes - Blanche-Benveniste identifie connaître, savoir, aimer, adorer et détester comme particulièrement fréquents. Par contraste, le Focus initial admet les noms spécifiques, définis ou indéfinis, entraîne des sujets clitiques et ne montre pas de restrictions lexicales particulières. En outre, bien que le Focus et la Dislocation à gauche sans reprise se retrouvent autant dans des propositions assertives qu'interrogatives:

14) a. Cécile, tu aimes pas?

b. Cécile que tu aimes pas?

c. dix centimes vous n'auriez pas? (Blanche-Benveniste, 1998 : 113)

le premier se retrouve uniquement dans les principales et assimilées, alors que comme les dislocations en général, la dislocation à gauche sans reprise se distribue également dans les subordonnées.

15) a. Je me demande si Cécile, tu (la) détestes.

b. ${ }^{*}$ Je me demande si Cécile (que) tu détestes. 
Les divergences concernant la présence du que cheville, l'impossibilité de la reprise pronominale et la distribution en principale s'expliquent par le mouvement du syntagme focalisé dans une projection de Focus en périphérie gauche, en principe non disponible dans les subordonnées. Ce mouvement syntaxique entraîne les effets de dépendance à longue distance, d'île syntaxique et de trous parasitiques (Abeillé, Godard \& Sabio, 2008a; Authier \& Haegeman, 2018). Ces observations permettent de compléter le tableau 1.

\begin{tabular}{|l|c|c|c|c|c|}
\hline & $\begin{array}{c}\text { Proposition } \\
\text { principale }\end{array}$ & $\begin{array}{c}\text { Interne à la } \\
\text { proposition }\end{array}$ & Position libre & Reprise & Topique \\
\hline Terme d'adresse & - & - & + & - & - \\
\hline Nominativus pendens & + & + & - & - & + \\
\hline Dislocation & - & + & + & + & + \\
\hline Dislocation gauche sans reprise & - & + & - & $/$ & + \\
\hline Focus initial & + & + & - & - & - \\
\hline
\end{tabular}

Tableau 1'. Éléments périphériques de la proposition conjuguée

On voit donc que la construction à Focus initial occupe une place distincte. Phénomène de proposition principale, sans liberté de position, sans possibilité de reprise, elle se distingue du nominativus pendens et de la dislocation par sa valeur présumée de... focalisation. Au regard des attestations suivantes, où le syntagme initial a intuitivement des interprétations très différentes, se pose la question de savoir quelle est la nature de cette valeur focalisante.

16) a. Une fortune ça m'a coûté! (Blanche-Benveniste, 2002: 111)

b. deux justificatifs de domicile il me faut (Blanche-Benveniste, 1998: 113)

c. Place Dauphine ça s'appelle (Blanche-Benveniste, 1998: 114)

d. ouvert 24 h sur 24 il y avait écrit (Blanche-Benveniste, 1998: 114)

C'est à cerner ces interprétations que s'attache la section qui suit.

\section{Valeur informationnelle}

La dernière colonne du tableau 1' dans la dernière section fait allusion à la valeur informationnelle typique des éléments périphériques. On admet en effet généralement que les syntagmes en périphérie gauche, 
au moins ceux en position de Topique ou de Focus, ont une valeur d'information nouvelle ou ancienne. La valeur de Focus correspond néanmoins à différents cas de figure, comme on le sait depuis Kiss (1988). C'est ce que nous articulons dans cette section.

Le cas prototypique est celui de Focus contrastif. Il s'agit du choix d'une entité en opposition à au moins une autre (voir Krifka, 2007 pour les différents sous-types de focus). Dans l'attestation ci-dessous, il est affirmé que les gens concernés mangent des pâtes et rien d'autre.

17) Rien que des pâtes ils mangent là-bas (Blanche-Benveniste, 1996 : 114)

Cette valeur est appuyée par l'expression restrictive rien que, les particules focalisantes restrictives et inclusives comme seulement et même y étant fréquentes selon Blanche-Benveniste.

Le contrastif n'est pas le seul cas concerné. Authier \& Haegeman (2018) souligne une valeur mirative. En se basant sur Cruschina (2009, 2011), ces derniers font valoir que le syntagme initial exprime une valeur «remarquable». C'est ce qu'exprime le syntagme initial dans l'attestation suivante.

18) Une fortune ça m'a coûté! (Blanche-Benveniste, 2002 : 111)

L'expression nominale fortune évoque du fait de sa signification de haut degré une valeur remarquable.

Les valeurs constrastives et miratives ne rendent cependant pas compte de tous les emplois. Considérez les deux exemples qui suivent:

19) a. et puis le beurre, tu me donnes (oral, Pohl, 52; Blanche-Beveniste, 1996: 113)

b. ouvert 24 h sur 24 il y avait écrit (Blanche-Benveniste, 1996: 114)

Ces attestations ne suggèrent pas un syntagme initial avec une valeur contrastée par rapport à une autre, ou qui soit particulièrement remarquable. Si elle est remarquable, elle ne l'est pas plus que si le syntagme était dans sa position postverbale ordinaire. C'est encore plus net dans les cas suivants:

20) a. mais sourds après on était tu vois (Blanche-Beveniste, $1996: 114$ )

b. le mec il l'a jeté de je sais pas combien d'étages - planté - écrasé il était (Blanche-Beveniste, 1996 : 114) 
où il n'est question ni de contraste ni de valeur particulièrement remarquable. Ainsi, ces exemples ne se paraphrasent pas naturellement par une clivée.

21) a.?? C'est sourd après qu'on était

b. ?? C'est écrasé qu'il était

La chose est notable, puisqu'elle suggère que la valeur du Focus initial n'est pas uniquement celle réputée contrastive de la clivée, mais peut évoquer une simple dimension présentative.

Cette situation nous oblige à trouver une façon alternative de décrire la valeur du Focus initial. Puisque cette configuration est censée mettre en jeu une information nouvelle, mais qu'elle semble dans les faits mettre en jeu des statuts informationnels divers, il nous a semblé utile d'explorer ces statuts systématiquement à l'aide d'élicitations, énoncés construits dont l'acceptabilité est vérifiée auprès de locuteurs natifs. Il s'agissait d'envisager des contextes où se retrouveraient les différentes combinaisons possibles de syntagme initial et de proposition avec pour l'un et l'autre une valeur d'information entièrement nouvelle, d'information inférable et/ou d'information explicitement ancienne. Ces contextes sont typiquement établis par les questions auxquelles un énoncé peut répondre. Soit la séquence Un nouveau boulot il a trouvé. Elle représenterait de l'information entièrement nouvelle dans une réponse à la question suivante:

22) Qu'est-ce qui se passe?

Cette même séquence constituerait de l'information inférable en réponse aux questions ci-dessous, par la relation entre occupation et boulot d'une part et chercher et trouver d'autre part.

23) Et Paul, ses occupations?

24) Et Paul, il cherche à s'en sortir?

25) Et Paul, il cherche des occupations?

Enfin, de l'information explicitement ancienne serait communiquée pour le syntagme focalisé, la proposition et toute la séquence à la suite de ces nouvelles questions.

26) Et Paul, son boulot?

27) Et Paul, il a trouvé de quoi s'en sortir?

28) Paul a trouvé un nouveau boulot? 
Les différentes combinaisons sont présentées dans les élicitations suivantes.

29) - Qu'est-ce qui se passe?

- ? *Un nouveau boulot il a trouvé, Paul.

30) - Et Paul, ses occupations?

- Un nouveau boulot il a trouvé.

31) - Et Paul, il cherche à s'en sortir?

- Un nouveau boulot il a trouvé.

32) - Et Paul, il cherche des occupations?

- Un nouveau boulot il a trouvé.

33) - Et Paul, il cherche un boulot?

- Oui, un nouveau boulot il a trouvé.

34) - Et Paul, son boulot?

- Un nouveau boulot il a trouvé.

35) - Et Paul, il a trouvé de quoi s'en sortir?

- Un nouveau boulot il a trouvé.

36) - Et Paul, il a trouvé des occupations?

- Un nouveau boulot il a trouvé.

37) - Paul a trouvé un nouveau boulot?

- Oui, un nouveau boulot il a trouvé.

Seul le contexte d'information entièrement nouvelle est incompatible avec le Focus initial, alors qu'une réponse de forme propositionnelle canonique est parfaitement naturelle.

38) - Qu'est-ce qui se passe?

- Paul a trouvé un nouveau boulot.

La distribution informationnelle des Focus initiaux est résumée par le tableau suivant:

\begin{tabular}{|l|l|c|c|c|}
\hline & \multicolumn{1}{|c|}{ Information } & Nouvelle & $\begin{array}{c}\text { Inférablement } \\
\text { ancienne }\end{array}$ & $\begin{array}{c}\text { Explicitement } \\
\text { ancienne }\end{array}$ \\
\hline Proposition & Nouvelle & $*$ & $(28)$ & $(32)$ \\
\hline & Inférablement ancienne & $(29)$ & $(30)$ & $(31)$ \\
\hline & Explicitement ancienne & $(33)$ & $(34)$ & (35) \\
\hline
\end{tabular}

Tableau 2. Valeur informationnelle du Focus initial dans des élicitations

La situation est différente avec les clivées, dont l'empan d'emploi est plus restreint. 
39) - Qu'est-ce qui se passe?

- ?* C'est un nouveau boulot qu'il a trouvé, Paul.

40) - Et Paul, ses occupations?

- ? C'est un nouveau boulot qu'il a trouvé.

41) - Et Paul, il cherche à s'en sortir?

- ? C'est un nouveau boulot qu'il a trouvé.

42) - Et Paul, il cherche des occupations?

- ? C'est un nouveau boulot qu'il a trouvé.

43) - Et Paul, il cherche un boulot?

- ? Oui, c'est un nouveau boulot qu'il a trouvé.

44) - Et Paul, son boulot?

- ? C'est un nouveau boulot qu'il a trouvé.

45) - Et Paul, il a trouvé de quoi s'en sortir?

- (?) C'est un nouveau boulot qu'il a trouvé.

46) - Et Paul, il a trouvé des occupations?

- C'est un nouveau boulot qu'il a trouvé.

47) - Paul a trouvé un nouveau boulot?

- ?? Oui, c'est un nouveau boulot qu'il a trouvé.

Ce qui manque dans les exemples construits précédents, c'est une dimension contrastive: en (44), la réponse semble plus naturelle car on peut comprendre boulot contrastant avec occupations.

Si les élicitations permettent de tester de façon symétrique les paramètres en jeu, elles ont le défaut que leur statut est parfois incertain par rapport à l'usage réel. En témoignent les jugements intermédiaires d'acceptabilité. C'est pourquoi nous nous tournons vers les attestations recueillies par Blanche Benveniste (1996 : 113ss) et par Abeillé, Godard \& Sabio $(2008 \mathrm{a} ; 2008 \mathrm{~b})$ pour évaluer le statut informationnel possible des configurations. La majorité des exemples ne sont pas fournis avec leur contexte antécédent. La conséquence en est qu'il est difficile d'évaluer le statut informationnel du terme focalisé et de la proposition. Ce sont les exemples avec un contexte antécédent permettant de juger du statut informationnel ancien ou nouveau à la fois du terme focalisé et de la proposition que nous avons recueillis dans ces deux études. Le statut informationnel du terme focalisé et de la proposition a été établi à partir d'un arbre de décision (repris de Larrivée, 2019): 
i. Has the phrase been explicitly mentioned in the previous discourse?

Yes $\rightarrow$ It is discourse-old No $\rightarrow$ Go to ii.

ii. Is the phrase referring by anaphor or deixis to a previously-mentioned phrase?

Yes $\rightarrow$ It is discourse-old $\quad$ No $\rightarrow$ Go to iii.

iii. Is the phrase used in a context that leads to infer it as discourse-old information based on a. previous discourse or b. world-knowledge?

Yes $\rightarrow$ It is discourse-old $\quad$ No $\rightarrow$ It is discourse-new.

Figure 1. Decision tree for information structure annotation

Cette démarche permet d'identifier la majorité des cas de figure.

a. Foyer nouveau et proposition nouvelle

48) a. et là tu sais qu'est-ce qu'il lui est arrivé - une antenne ils lui ont jeté sur la tête (oral, Nord 40,3) (Blanche Benveniste, 1996 : 113) b. le mec il l'a jeté de je sais pas combien d'étages - planté - écrasé il était (Nord 89, 5) (Blanche Benveniste, 1996 : 114)

b. Foyer inférablement ancien et proposition nouvelle

49) a. J'ai écrit dans le journal local d'Aire-sur-la-Lys je me rappelle plus maintenant ah L'Echo de la Lys // ça s'appelait je crois bien. [Corpaix] (Blanche Benveniste, 1996)

b. Loc1: c'est quoi la région que vous préférez + Loc2 : [...] moi j'aime bien la Bretagne quoi + j'aime bien ma Bretagne + j'aurais du mal à aller ailleurs quoi + l'Auvergne aussi ben c'est vrai que c'est c'est vert aussi hein c'est beau c'est

Loc1: Paris

Loc2: Paris je connais pas [cx 1, CRFP : QUI-R00PRI001] (Abeillé, Godard \& Sabio, 2008b, ex. (26))

c. Foyer inférablement ancien et proposition inférablement ancienne

50) Loc1: Quelle est la matière qui t'a le moins plu et pourquoi Loc2: euh + la grammaire j'ai pas du tout apprécié parce que en fait j'étais pas très bonne (Abeillé, Godard \& Sabio, 2008b, ex. (24b))

d. Foyer inférablement ancien et proposition explicitement ancienne

51) a. ils ont pas gardé tout le monde - pourquoi tes parents ils les ont gardés (L955-3-35, 3, 1) (Blanche Benveniste, 1996 : 116)

b. et je lui apprenais à lire à écrire - enfin tout ce que je savais - je lui ai appris (Baral 259, 3) (Blanche Benveniste, 1996 : 116) 
e. Foyer explicitement ancien et proposition nouvelle

52) J'ai commencé à sept ans // sept ans et demi huit ans je sais plus exactement quatre- vingt-s huit ans je devais avoir [CFRP: BORR00PRI001] (Abeillé, Godard \& Sabio, 2008b, ex. (24b))

f. Foyer explicitement ancien et proposition explicitement ancienne

53) a. depuis qu'il y a les grèves - Jacques Chirac on le voit plus tu l'as pas vu une seule fois aux informations - pas une fois tu l'as vu (Nord 40, 3) (Blanche Benveniste, 1996: 113)

b. Denise mon père il va m'acheter un petit mouton - un petit mouton il va m'acheter (Agenet Gr3, 6) (Blanche Benveniste, 1996: 116)

Soit, en tableau:

\begin{tabular}{|l|l|l|c|c|}
\hline & \multicolumn{1}{|c|}{ Information } & Nouvelle & $\begin{array}{c}\text { Inférablement } \\
\text { ancienne }\end{array}$ & $\begin{array}{c}\text { Explicitement } \\
\text { ancienne }\end{array}$ \\
\hline Proposition & Nouvelle & $(48)$ & $(49)$ & $(52)$ \\
\hline & Inférablement ancienne & & $(50)$ & \\
\hline & Explicitement ancienne & & $(51)$ & (53) \\
\hline
\end{tabular}

Tableau 3. Valeur informationnelle du Focus initial dans des attestations

Manquent les cas suivants:

h. Foyer nouveau et proposition inférablement ancienne

54) - Combien ça coûte?

- 100 francs j’ai payé.

i. Foyer nouveau et proposition explicitement ancienne

55) - Combien ça coûte?

- 100 francs ça coûte.

j. Foyer explicitement ancien et proposition inférablement ancienne

56) - Ça coûte 100 francs?

- 100 j'ai payé.

\begin{tabular}{|c|c|c|c|c|}
\hline & & Foyer & & \\
\hline & Information & Nouvelle & $\begin{array}{l}\text { Inférablement } \\
\text { ancienne }\end{array}$ & $\begin{array}{l}\text { Explicitement } \\
\text { ancienne }\end{array}$ \\
\hline \multirow[t]{3}{*}{ Proposition } & Nouvelle & (48) & (49) & (52) \\
\hline & Inférablement ancienne & (54) & (50) & (56) \\
\hline & Explicitement ancienne & (55) & (51) & (53) \\
\hline
\end{tabular}

Tableau 3'. Valeur informationnelle du Focus initial dans des attestations, complété 
Ce que montre cette section, c'est que la valeur souvent élusive des configurations marquées comme le Focus initial peut être cernée grâce à une évaluation de leur dimension informationnelle. On trouvera un encouragement à cette démarche dans le fait que convergent les résultats des données élicitées et attestées. Les résultats sont résumés dans la section finale, qui les situe dans la problématique générale de la fréquence de la configuration.

\section{Structure et fréquence}

Ce travail a montré que le français a une construction à Focus initial, comme l'anglais ou l'italien:

57) Marina ha invitato (Bianchi, 2013: 193)

Marina (il) a invité

58) Macadamia nuts I think they're called (Prince, $1981: 250$ )

Noix de Macadamia je crois qu'elles s'appellent

Syntaxiquement, cette construction met en jeu le mouvement d'un syntagme en position initiale de Focus. Par conséquent, elle est toujours en principale, y occupe une position fixe, et sa reprise par un pronom dans la proposition est exclue. La palette interprétative de cette construction est vaste. Elle communique des Focus contrastifs (57), miratifs (58), mais aussi présentatifs (59).

59) Rien que des pâtes ils mangent là-bas (Blanche-Benveniste, 1996:

60) Une fortune ça m'a coûté! (Blanche-Benveniste, 2002: 111)

61) et puis le beurre, tu me donnes (oral, Pohl 1976: 52; BlancheBeveniste, 1996 : 113)

C'est ce que montre l'étude de la valeur informationnelle de la configuration à partir à la fois d'attestations et d'élicitations, qui sert à établir que la configuration a un empan interprétatif plus large que celui des clivées par exemples. Les jalons posés dans cette étude permettront, on ose l'espérer, de préciser les contours interprétatifs, souvent incertains, des configurations informationnelles.

L'étude a été poursuivie à partir du constat intuitif que la construction est rare en français, alors qu'elle est peu fréquente en anglais et relativement commune en italien. Il n'y a guère d'études quantitatives des configurations informationnelles à travers les langues. 
L'étude des clivées dans des données orales formelles par Dufter (2008) suggère que le français en fait une utilisation beaucoup plus importante que d'autres langues; elles sont aussi fréquentes qu'en portugais, un tiers plus fréquentes qu'en italien et en espagnol, et quatre fois plus fréquentes qu'en allemand. Comme mentionné auparavant, les clivées représentent 3\% des propositions en français vernaculaire, alors qu'en extrapolant à partir de Garassino (2016), on les trouve à un demi pour cent pour l'italien, et à un quart pour l'anglais. Je ne connais pas d'étude quantitative de la prévalence du Focus initial (qui comme les clivées varie à travers les langues romanes, Leonetti, 2017; sur l'occitan où le Focus initial serait fréquent, voir Faure, 2013). La lecture des échanges vernaculaires les plus spontanés (échanges apéro) rendus disponibles par le corpus CLAPI donne deux occurrences sur 900 propositions, à un taux de $0,11 \%$. Ce taux, qui est celui des nominativus pendens (Larrivée, 2015), contraste fortement avec celui de la clivée, et des dislocations. De là, il est tentant d'attribuer la rareté des Focus initiaux à la fréquence des clivées, d'autant que ces configurations ont des propriétés convergentes. Leurs divergences tiennent à la syntaxe (la clivée n'étant pas limitée aux principales) et à l'interprétation (la clivée semblant avoir un empan interprétatif plus étroit).

L'idée de la prégnance d'une variable clivée aux dépens du Focus contrastif ne résout pas la question de savoir pourquoi cette première variable est plus prégnante. Un autre facteur décisif est le statut prosodique des syntagmes préverbaux du français. Comme l'a souligné Lambrecht (1994), dans cette langue, l'accent d'insistance est pratiquement agrammatical en position préverbale. Cela renforce l'argumentation d'Hinterholzl (2018) selon qui chaque langue fait des choix différents en matière de marquage informationnel, ce qui a une répercussion sur la disponibilité des autres marquages. Par conséquent, bien que toutes les grandes catégories de syntaxe soient disponibles dans toutes les langues, leur mode de réalisation peut être raisonnablement contraint par les conditions d'interface imposées par des idiomes particuliers.

\section{Conclusion}

Cet article s'est intéressé à la construction à Focus initial en français, illustrée en (9). Après un survol des propriétés formelles de 
cette configuration, deux dimensions en ont été explorées. La première est celle de la valeur informationnelle du syntagme initial. Le terme de focus laisserait attendre une valeur d'opposition, qui n'est pas la seule attestée cependant. En effet, on a vu, à partir d'un arbre de décision et d'un examen systématique à la fois d'attestations et d'élicitations, que tous les statuts informationnels peuvent être endossés par le syntagme initial comme par la proposition. L'autre résultat nouveau de cette étude est la formulation d'une explication de la faible fréquence de la configuration. La rareté en français d'un Focus initial plus répandu dans d'autres langues romances s'expliquerait par la difficulté d'y avoir un accent d'insistance préverbal. Cela pourrait expliquer, par compensation la plus grande fréquence de la clivée en français. Bien sûr, ces propositions nouvelles pourront être testées plus avant quand sera mis au jour un plus grand nombre de données attestées, dans des corpus de vernaculaire annotés syntaxiquement par exemple.

\section{Bibliographie}

ABEILlE A., GODARD D. \& SABIO F (2008a), Two types of NP preposing in French, in Stefan Müller (ed.), Proceedings of the 15th international conference on HPSG. Stanford, CSLI Publications, 306-324.

ABEILlE A., GODARD D. \& SABIO F. (2008b), Deux constructions à SN antéposé en français, in Durand B., Habert B. \& Laks B. (éds), Actes CMLF 2008, 2361-2376.

AUTHIER J.-M. \& HAEGEMAN L. (2018), The syntax of mirative Focus Fronting: Evidence from French, in Arteaga D. L. (ed.), Contribution of Romance languages to current linguistic theory, Cham, Springer, 39-64.

BIANCHI V. (2013), On focus movement in Italian, in Camacho Taboada M. V., Jiménez-Fernández Á., Martín-González J. \& Reyes-Tejedor M. (eds), Information Structure and agreement, Amsterdam, Benjamins, 193-216.

BLANCHE-BENVENISTE C. (1996), Trois remarques sur l'ordre des mots dans la langue parlée, Langue française 111, 109-117.

BLANCHE-BENVENISTE C. (2002), Macro-syntaxe et micro-syntaxe: les dispositifs de la rection verbale, in Andersen H. L. \& Nølke H. (éds), Macro-syntaxe et macro-sémantique, Berne, Peter Lang, 95-118. 
BLASCO M. (1995), Dislocation et thématisation en français parlé, Revue sur le français parlé 13, 46-63.

CRUSCHINA S. (2009), The syntactic role of discourse-related features, Cambridge Occasional Papers in Linguistics 5, 15-30.

CRUSCHINA S. (2011), Discourse-Related Features and Functional Projections, Oxford, Oxford University Press.

DE CAT C. (2008), French dislocation. Interpretation, syntax, acquisition, New York et Oxford, Oxford University Press.

DE CESARE A.-M. (2017), Cleft constructions, in Dufter A. \& Stark E. (eds.), Manual of Romance Morphosyntax and Syntax, Berlin, De Gruyter Mouton, 536-568.

DEULOFEU H. J. (1977), La syntaxe et les constructions binaires, Recherches sur le français parlé 1, 30-61.

DUFTER A. (2008), Clefting and discourse organization: Comparing Germanic and Romance, in Dufter A. \& Jacob D. (eds.), Focus and background in Romance languages, Amsterdam, Benjamins, $83-121$.

FAURE R. \& OLIVIERI M. (2013), Stratégie de topicalisation en occitan, Corpus 12, 231-270.

GARASSINO D. (2016), Using cleft sentences in Italian and English: A multifactorial analysis, in de Cesare A.-M. \& Garassino D. (eds.), Current Issues in Italian, Romance and Germanic noncanonical Word Orders: Syntax-Information StructureDiscourse Organization, Berne, Peter Lang.

GROUPE DE FRIBOURG (2012), Grammaire de la période, Berne, Peter Lang.

HINTERHOLZL R. (2018), Prominence, accent and Information Structure, Linguistik online 88, 63-84.

KISS K. É. (1988), Identificational focus versus information focus, Language 74: 2, 245-273.

KRIFKA M. (2007), Basic notions of Information Structure, in C. Féry, G. Fanselow \& M. Krifka (eds.), Interdisciplinary Studies on Information Structure, Universitätsverlag Potsdam, 61-96.

LAGORGETTE D. (2003), Termes d'adresse, insulte et notion de détachement en diachronie: quels critères d'analyse pour la fonction d'adresse?, Cahiers de Praxématique 40, 43-70.

LAMBRECHT K. (1987), Presentational cleft constructions in spoken French, in Haiman J. \& Thompson S. A. (eds.), Clause combining in grammar and discourse, Amsterdam, Benjamins, 135-179. 
LAMBRECHT K. (1994), Information Structure and sentence form: Topic, focus, and the mental representation of discourse referents, Cambridge, Cambridge University Press.

LARRIVÉE P. (2015), Ordre des mots de la proposition conjuguée, Fiche Fracov, <http://www.univ-paris3.fr/index-des-fiches-227311. kjsp?RH=1373703153287>.

LARRIVÉE P. (2019), To be or not to be informational: Preverbal complements in Medieval French V2 configurations, Glossa 4: 1.

LEGENDRE G. \& CULBERTSON J. (2008), Qu'en est-il des clitiques sujet en français oral contemporain?, in Durand J. Habert B., Laks B. (éds.), Congrès Mondial de Linguistique Française CMLF 2008, Paris, Institut de Linguistique Française, 26632674. <https://doi.org/10.1051/cmlf08308>.

LEONETTI M. (2017), Basic Word Order, in Dufter A. \& Stark E. (eds), Manual of Romance morphosyntax and syntax, Berlin, De Gruyter Mouton.

LE QUERLER N. (2003), Le nominativus pendens en français, Cahiers de Praxématique 40, 149-166.

MEISNER C. (2010), A Corpus Analysis of Intra- and Extralinguistic Factors triggering ne-Deletion in Phonic French, in Neveu F., Muni Toke V., Durand J., Klingler T., Mondada L. \& Prevost S. (éds.), Congrès Mondial de Linguistique Française - CMLF 2010, Paris, Institut de Linguistique Française.

MISSIRE R. \& ROUAYRENC C. (2014), Contribution à la description du préambule en français oral spontané, in Missire R. (éd.), Approches sémantiques de l'oral, Louvain, Academia, 165-180.

NOLDA A. (2013), Topics detached to the Left: On 'Left Dislocation', 'Hanging Topic', and related constructions in German, in Shaer B., Frey W. \& Maienborn C. (eds), Proceedings of the Dislocated Elements Workshop, ZAS Berlin, November 2003, Berlin, ZAS, 423-448.

POHL J. (1976), Les constructions «about» - pronom sujet dans le français contemporain, in Boudreault M. \& Möhren F. (éds), Actes du XIII ${ }^{e}$ Congrès international de linguistique et philologie romanes, vol. 1, Québec : Presses de l'Université Laval, 499-514.

POHL J. (1984), Documents pour servir à l'étude des phrases du type Les fleurs, j'aime, Romanistisches Jahrbuch 35: 1, 36-58.

PRINCE E. (1981), Topicalization, Focus-movement, and Yiddishmovement: A pragmatic differentiation, Proceedings of the Seventh Annual Meeting of the Berkeley Linguistics Society, 249-264. 
QUIGNARD M., URSI B., ROSSI-GENSANE N., ANDRE V., BALDAUF-QUILLIATRE H., ETIENNE C., PLANTIN C., TRAVERSO V. (2016), Une méthode instrumentée pour l'analyse multidimensionnelle des tonalités émotionnelles dans l'interaction, in Neveu F., Bergounioux G., Côté M.-H., Fournier J.-M., Hriba L. et Prévost S. (éds), Congrès Mondial de Linguistique Française - CMLF 2016, Paris, Institut de Linguistique Française. <https://doi.org/10.1051/ shsconf/20162715004>.

RIZZI L. (1997), The structure of the left periphery, in Haegeman L. (ed.). Elements of grammar, Dordrecht, Kluwer, 281-337.

ROWLETT P. (1998), The Grammar of French, Oxford, Oxford University Press.

SABIO F. (1995), Micro-syntaxe et macro-syntaxe: l'exemple des « compléments antéposés» en français, Recherches sur le français parlé 13, 111-155.

STEMPEL W.-D. (1981), "L'amour, elle appelle ça», "L'amour tu ne connais pas», in ROHRER C. (éd.), Studia Linguistica in honorem Eugenio Coseriu, vol. VI : Grammatik, Berlin: De Gruyter, 351-367.

TANGUY N. (2010), Focalisation averbale vs focalisation verbale en français parlé: Le cas des constructions binaires, Discours 6 .

WARD G. \& BIRNER B. (2011), Discourse effects of word order variation, in von Heusinger K., Maienborn C. \& Portner P. (eds), Semantics: An international Handbook of natural language meaning, volume 2, Berlin \& Boston: Mouton de Gruyter, 1934-1962. 